\title{
1 An investigation of infrasound propagation over mountain ranges
}

${ }^{1}$ Commissariat à l'Énergie Atomique et aux Énergies Alternatives, 91297 Arpajon, France

${ }_{4} \quad 2$ Laboratoire de Météorologie Dynamique, École Normale Supérieure, 75231 Paris, France

${ }_{5}{ }^{a}$ Also at: Centre de Mathématiques et Leurs Applications, École Normale Supérieure Paris6 Saclay, 94235 Cachan, France. Electronic mail: christophe.millet@cea.fr. 


\section{Abstract}

Linear theory is used to analyze trapping of infrasound within the lower tropospheric waveguide during propagation above a mountain range. Atmospheric flow produced by the mountains is predicted by a nonlinear mountain gravity wave model. For the infrasound component, we solve the wave equation under the effective sound speed approximation using both a finite difference method and a WKB approach. It is shown that in realistic configurations, the mountain waves can deeply perturb the low level waveguide, which leads to significant acoustic dispersion. To interpret these results each acoustic mode is tracked separately as the horizontal distance increases. It is shown that during statically stable situations, situations that are common during night over land in winter, the mountain waves induce a strong Foehn effect downstream, which shrinks the waveguide significantly. This yields a new form of infrasound absorption, that can largely outweigh the direct effect the mountain induces on the low level waveguide. For the opposite case, when the low level flow is less statically stable (situations that are more common during day in summer), mountain wave dynamics do not produce dramatic responses downstream. It may even favor the passage of infrasound and mitigate the direct effect of the obstacle. 
Damiens, Millet and Lott, J. Acoust. Soc. Am., p. 3

\section{Introduction}

Infrasound, which is defined as sound waves that are lower in frequency than $20 \mathrm{~Hz}$, is characterized by an ability to travel over long horizontal distances in the atmosphere. This is related to the fact that the wind and temperature strongly vary with altitude, providing multiple ducts in which infrasound can propagate efficiently ${ }^{20}$. Although an important duct is potentially in the lower thermosphere, as a result of the steep increase in temperature (e.g. above $90 \mathrm{~km}$ ), the decrease in mean density produces substantial absorption coefficients there $^{42}$. For this reason, the most efficient ducts are often within the middle atmosphere, e.g. above the tropopause at around $20 \mathrm{~km}$ and below the mesopause at $90 \mathrm{~km}$. At lower altitudes, infrasound can also be trapped within tropospheric waveguides over distances that may reach several hundred of kilometers, at least when the weather conditions permit ${ }^{43 ; 31}$. However in this case, the wave interacts with the ground surface much more than wave refracting higher in the atmosphere and topographic features produce quantifiable effects on the recorded data ${ }^{30}$. Furthermore, at these altitudes the absorption coefficient is small and thus, it is neglected in most practical applications.

Although much less studied, the propagation of infrasound over distances of a few tens of kilometers can be controlled by a planetary boundary layer duct ${ }^{11 ; 45}$, which is a region of approximately $1 \mathrm{~km}$ depth in which the boundary effects are reflected in the flow ${ }^{16}$. For these relatively short propagation ranges, the upward refraction at higher altitudes (around 
42

and above the tropopause) can be ignored ${ }^{45}$ and the acoustic field can be described by a modal expansion involving a few modes. For completeness, it is important to note that the absorption properties of ground play a significant role ${ }^{4}$, in the sense that vegetationcovered land absorbs more energy than bare-ground for instance. We know that some modes are sensitive to such absorptions ${ }^{44 ; 47}$ but we will not include these effects here, essentially because a comprehensive theory of acoustic propagation which accounts for both absorption by vegetation and turbulence is lacking.

The common approach to calculate infrasound propagation in the atmosphere consists in solving the acoustic equation in a given background atmospheric state that varies with altitude and horizontal distance. This approach captures the most significant ducts, but sometimes it fails in predicting important arrivals ${ }^{17 ; 3 ; 34}$. The reason is that the atmospheric specifications, that are issued from operational numerical weather forecasts (e.g., provided by the ECMWF Integrated Forecast System or the NOAA Global Forecast System) or atmospheric climate reanalysis (e.g., ERA-Interim or NASA Modern Era Retrospective Reanalysis for Research and Applications), are associated with spatial resolutions that are much larger than the typical infrasound wavelength. These products therefore fail in representing important small-scale atmospheric fluctuations that can substantially modify the larger-scale ducts $^{6}$, especially for borderline ducts that barely return sound to the ground. The statistics of these fluctuations, however, are poorly understood, whereas their knowledge is required 
61

for infrasound propagation modeling. For instance, Chunchuzov et al. ${ }^{12}$ have shown the need to introduce random atmospheric perturbations to adequately represent the acoustic properties of the boundary layer, but in their work the sources of perturbations are not specified. As in the troposphere the (unresolved) fluctuations are mainly produced by mountains ${ }^{18}$, the contribution of these mountains to infrasound propagation remains an important open question.

In a first attempt to incorporate topography effects in acoustic propagation, highresolution terrain models have been used to represent the lower boundary by a sequence of up and down stair steps ${ }^{2 ; 30}$. In this approach, mountains directly modify the altitude of the lower boundary of the troposphere, which affects the acoustic cut-off frequencies of the corresponding ducts. This can be viewed as applying a "mask" onto the atmospheric specifications, and ignoring the direct influence of the mountain ridges on the local wind and temperature fields. This is an extremely serious limitation, given that mountains can dynamically produce very intense phenomena, like downslope winds, Foehn, or trapped lee waves $^{40 ; 15}$. As an illustration, it is worth mentioning that even small "mountains", with elevations of a several hundred meters, can develop substantial winds and temperature disturbances, depending on the incoming flow structure ${ }^{15}$.

There are two primary objectives in the present investigation. The first is to compare the results of the "mask" approximation to that obtained with a wind model that captures 
the interaction between the topography and the boundary layer. The second objective is to examine the physical mechanisms that cause a low-level acoustic duct to be affected and eventually destroyed by mountain-induced disturbances. Here we use the mountain flow model described by Lott $^{29}$. With respect to our first objective, this model involves a nonlinear boundary condition, i.e. it includes an obstacle that penetrates inside the low level waveguide and reduces its depth, an effect that potentially recovers the classical "mask" technique. It is worth while to point out that the model also predicts a mountain wave field, which compares in amplitude to the background winds and temperature variations responsible for the waveguide. This inherently affects the trapped acoustic modes, yielding highly dispersed signals as well as irreversible absorption of the acoustic wave passing over the ridge.

The paper is organized as follows. In section II, the mountain wave model is described and the dominant features of the mountain wave field are discussed in terms of dimensionless Richardson and Froude numbers. The effect of mountain wave disturbances on the acoustic field $p(x, z)$ is then considered in section III, using a classical range-dependent normal mode approach $^{24}$ to account for flow changes along the source-receiver distance $x$. To make the absorptive properties more transparent, the acoustic modes are also obtained using a WKB approximation. In section IV, it is found that the interaction between the mountain flow and the acoustic field gives rise to attenuation or amplification of ground-based signals, 
99

depending on the statical stability of the boundary layer flow. The characteristics of the perturbed acoustic modes such as phase velocities, attenuations, and wave structures in the $(x, z)$-plane are provided and discussed. In section $\mathrm{V}$, the downstream attenuation is systematically evaluated in terms of dimensionless numbers that control the mountain flow dynamics. Importantly, it is found that in near-neutral conditions, the mountain wave dynamics can favor infrasound propagation above the mountain, mitigating the direct effect of the obstacle.

\section{Atmospheric mountain flow model}

\section{A. Formalism}

Mountain waves, that occur when a stably stratified flow is forced by an obstacle, are often standing or nearly so, at least to the extent that the upstream environmental conditions are stationary. They can accompany Foehn wind conditions that are characterized by warm and dry downslope winds on the lee side of mountains ${ }^{37}$. In the present study, we use the mountain wave model developed by Lott ${ }^{29}$, which is adapted from Long ${ }^{27}$ 's model to incoming shear flows that varies with altitude. Comparisons with nonlinear simulations ${ }^{13}$ demonstrated that this model is well-suited for capturing realistic features of mountain flow dynamics.

In the present study, the mean state consists of an isothermal atmosphere, at temper- 
Damiens, Millet and Lott, J. Acoust. Soc. Am., p. 8

ature $T_{0}$, in the presence of a background wind $U(z)$ which is assumed to be in the shape of an hyperbolic tangent function. This representation is appropriate to describe the planetary boundary layers ${ }^{8 ; 10}$ and can even be used to initialize mesoscale models ${ }^{35}$. Although such a profile can occur during strong stratification or above the lowest maximum of the wind speed $^{32}$, there are many other semi-empirical models that adequately describe the wind shear. Here, the profile is used to mimic the incoming boundary layer, so that stationary gravity waves can be generated through interaction with the mountain, as observational evidence $^{9 ; 41}$ suggest. Specifically, the mean flow is given by

$$
T(z)=T_{0}, \quad U(z)=U_{0} \tanh (z / \delta),
$$

where $\delta$ is the boundary layer thickness, $U_{0}$ denotes the maximum wind speed over the mountain, and $z$ is the height, which is here typically smaller than $5 \mathrm{~km}$. The thermodynamic sound speed $c_{0}$ is given by $c_{0}^{2}=\gamma R T_{0}$, where $\gamma$ is the ratio of specific heats and $R$ is the specific gas constant for dry air. Hence, in an isothermal atmospheric boundary layer, the sound speed is constant. Using the ideal gas law and hydrostatic balance we know that in an isothermal atmosphere the background pressure and density vary as $\exp \left(-g z / R T_{0}\right)$, and the background potential temperature $\theta$ is related to the Brunt-Väisälä frequency ${ }^{20} N$ through

$$
N^{2}=\frac{g}{\theta} \frac{d \theta}{d z}=\frac{\gamma-1}{\gamma} \frac{g^{2}}{R T_{0}}
$$

where $g$ is the gravitational constant. Solving (2), we observe that $\theta$ varies as $\exp \left(N^{2} z / g\right)$, 
Damiens, Millet and Lott, J. Acoust. Soc. Am., p. 9

which provides the stratification needed for internal gravity waves to develop.

Now, given this stratification (through $N$ ), it is conventional to neglect the vertical changes of background density for relatively small $\delta$ (typically less than $1 \mathrm{~km}$ ). This is the classical Boussinesq approximation, that we can adopt here because our focus lies on the low level waveguide. Within the framework of the above hypothesis, and following previous works $^{29}$, the vertical perturbation in the velocity is given by the (inverse) Fourier transform

$$
w^{\prime}(x, z)=\int_{\mathbb{R}} f(k) \hat{w}_{c}(k, z) \mathrm{e}^{i k x} \mathrm{~d} k
$$

where $f(k)$ is an amplitude function that depends on the wavenumber $k$, and $\hat{w}_{c}(k, z)$ is a canonical solution satisfying the Taylor-Goldstein equation

$$
\frac{\mathrm{d}^{2} \hat{w}_{c}}{\mathrm{~d} z^{2}}+\left[\frac{N^{2}}{U^{2}}-\frac{U_{z z}}{U}-k^{2}\right] \hat{w}_{c}=0
$$

with the condition $\hat{w}_{c}(k, z) \sim \mathrm{e}^{-\lambda(k) z}$, as $z \rightarrow \infty$, and where the square-root function $\lambda$ is defined by

$$
\lambda(k)=\left[k^{2}-\frac{N^{2}}{U_{0}^{2}}\right]^{\frac{1}{2}} .
$$

In order that the boundedness or outgoing-wave condition be satisfied as $z \rightarrow \infty$, the branch cuts of $\lambda$ are inserted such that we have $\hat{w}_{c}(k, z) \sim \mathrm{e}^{i \epsilon \lambda(k) z}$ where $\epsilon=\operatorname{sign}(k)$ is to ensure upward propagation for $|k|<N / U_{0}$.

The boundary condition at $z \rightarrow \infty$ and the choice of branch cuts allow the solution of (4) to be expressed in terms of hypergeometric functions. A dynamically consistent horizontal 
velocity field $u^{\prime}$ can be obtained in spectral space using a polarization relation ${ }^{18}$. The amplitude $f(k)$ is then determined through inversion of the "free-slip" nonlinear boundary condition

$$
w^{\prime}(x, h(x))=\left[U(h)+u^{\prime}(x, h(x))\right] \frac{d h}{d x},
$$

with the witch of Agnesi profile

$$
h(x)=\frac{H}{1+\frac{x^{2}}{2 L^{2}}},
$$

where $H$ is the ridge top height and $L$ is a characteristic length scale. Application of the Fourier transform to $(7)$ leads to $\hat{h}(k)=H L \mathrm{e}^{-k \sqrt{2} L} / \sqrt{2}$, which implies that the dominant horizontal wavelength is given by $k=1 / \sqrt{2} L$. In the following, this profile will be centred at $x_{0}=25 \mathrm{~km}$ and we will use $h(x)$ instead of $h\left(x-x_{0}\right)$ for notational conciseness.

To describe the flow response, it is also worthwhile to use the three dimensionless parameters

$$
J=\frac{N^{2} \delta^{2}}{U_{0}^{2}}, \quad H_{N}=\frac{N H}{U_{0}}, \quad \text { and } \quad F=\frac{N L}{U_{0}} .
$$

While the Richardson number $J$ measures the background flow stability ${ }^{33 ; 23}$, the other parameters are related to the shape of the mountain. The parameter $H_{N}$ is a dimensionless mountain height that measures the degree of nonlinearity in the flow response ${ }^{39}$. The classical Froude number $F$ compares the advective time-scale to cross the ridge and the buoyancy oscillation time-scale. This last parameter measures the significance of non-hydrostatic effects $^{36}$. In the following we will fix $N, U_{0}$ and $L$ such that $F=10 \gg 1$, a value that 
164

guarantees that no substantial trapped lee waves are forced. We will vary the boundary layer depth $\delta$ and/or the mountain height $H$.

\section{B. Effective sound speed disturbances}

Following Waxler ${ }^{44}$ we next use the effective sound speed approximation ${ }^{19}$, in which the component of the horizontal wind speed in the direction of propagation is added to the thermodynamic sound speed. For an isothermal atmosphere, with a varying background wind $U$, this approximation yields $c(z)=c_{0}+U(z)$ and thus, ducting is only due to the change in altitude of $U$. Hence, in presence of temperature and wind fluctuations, the perturbed effective sound speed is given by

$$
c_{0} \sqrt{1+\frac{T^{\prime}}{T_{0}}}+U+u^{\prime}
$$

where the temperature and the horizontal wind perturbations, which are denoted by $T^{\prime}$ and $u^{\prime}$, respectively, are obtained from the vertical velocity $w^{\prime}$ using polarization relations ${ }^{18}$.

In order to illustrate how mountain waves can perturb the background state, the mountain wave model described in section II.A is used with parameters that are representative of the lower troposphere. Here, and in the following, we consider a boundary layer flow at $U_{0}=10 \mathrm{~m} \cdot \mathrm{s}^{-1}$ in a stratified medium characterized by $N=\sqrt{2} \cdot 10^{-2} \mathrm{~s}^{-1}$, and take $L=10 \mathrm{~km}$ to enforce $F=10$. For illustrative purposes, the height of the mountain and the boundary layer thickness are fixed to $H=350 \mathrm{~m}$ and $\delta=860 \mathrm{~m}$, respectively. For these parameters, 

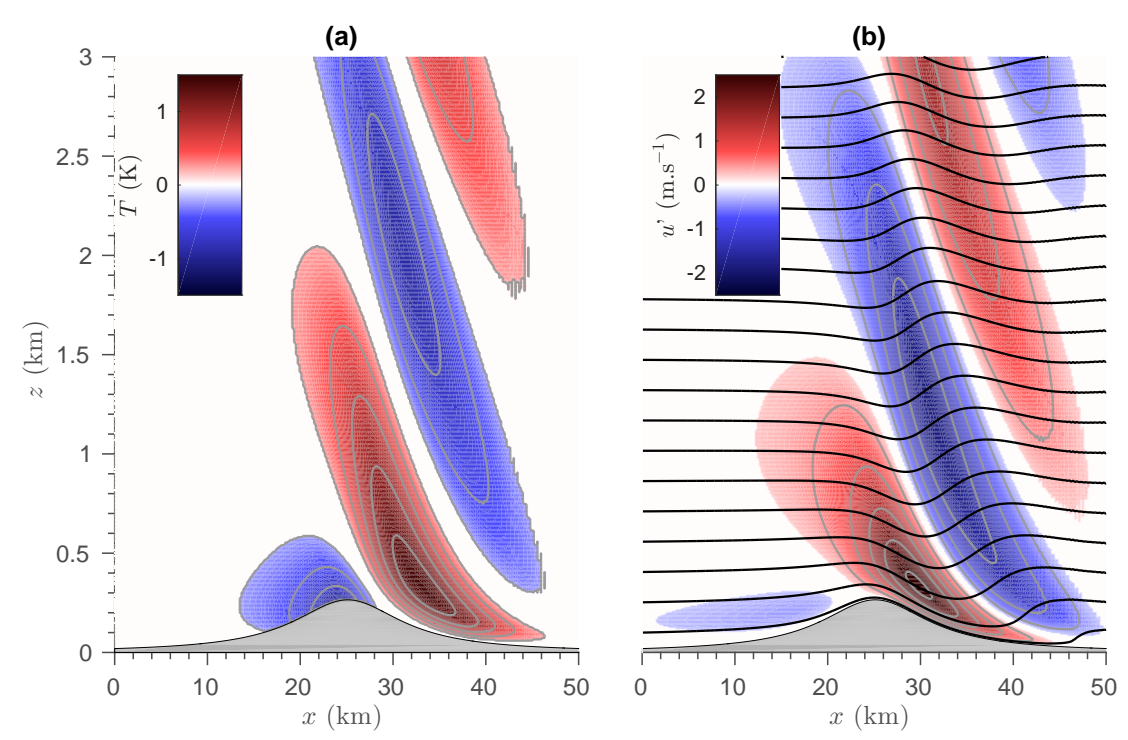

Figure 1: (a) Temperature fluctuations $T^{\prime}$ and (b) horizontal wind fluctuations $u^{\prime}$ resulting from interaction between a mountain and an incoming boundary layer. The streamlines are given by black lines superimposed to the wind fluctuations. The dimensionless parameters used are $H_{N}=0.5$ and $J=1.5$. The mountain is represented in gray. we obtain $J=1.5$, which corresponds to a moderately stable situation. Finally, the dimensionless value $H_{N}=0.5$ is sufficiently small to guarantee that the near-linear mountain flow theory applies and produces realistic downslope winds and Foehn.

Figure 1a and 1b show the temperature and wind fluctuations produced by the mountain 

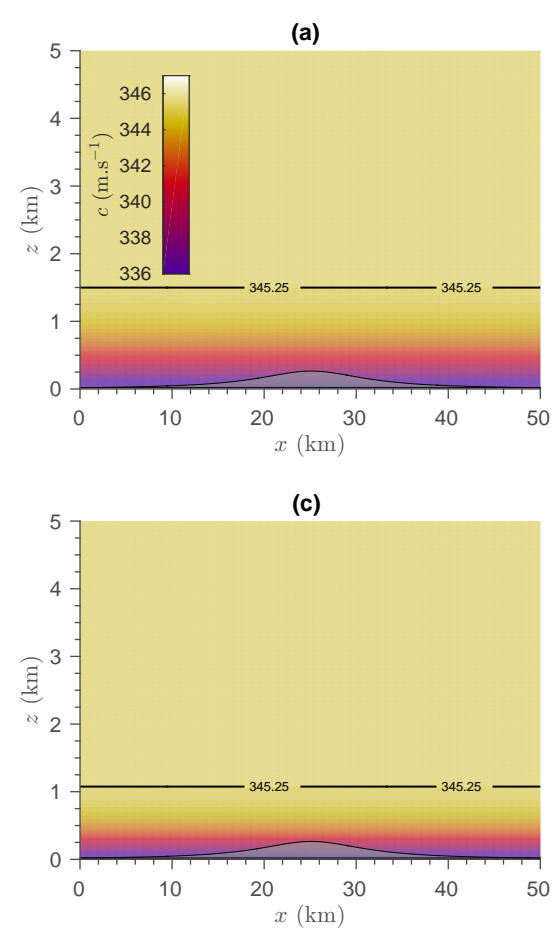

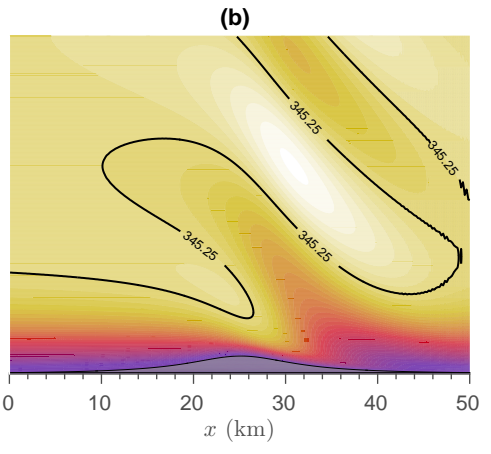

(d)

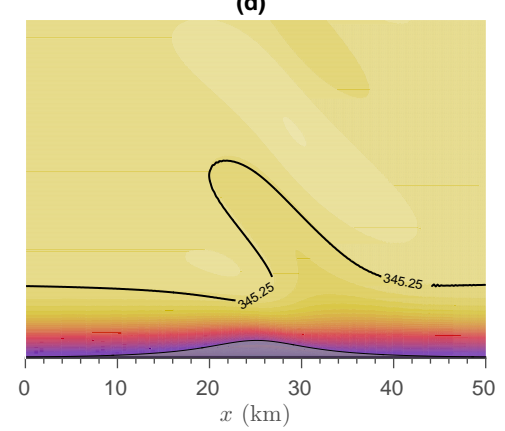

Figure 2: Effective sound speed field without $(\mathrm{a}, \mathrm{c})$ and with $(\mathrm{b}, \mathrm{d})$ interaction between a mountain and an incoming boundary layer. The dimensionless parameters used are $H_{N}=0.5$ (a,b,c,d) and $J=1.5(\mathrm{a}, \mathrm{b})$ or $J=0.5(\mathrm{c}, \mathrm{d})$.

windstorms. The streamlines are represented in figure $1 \mathrm{~b}$ to illustrate the so-called isentropic drawdown mechanism often used to explain Foehn. In this dry mechanism the Foehn results 
and V. In figure 2a,c we just keep the incident waveguide unaltered and chopped it by the mountain height for $J=1.5$ (figure 2a) and $J=0.5$ (figure 2c). This is representative of the "mask" technique used in the literature ${ }^{2}$, and to which we will systematically compare our results to in the following. From figure 2a we can expect its effect to be substantial since this mask potentially excludes from trapping all the waves with phase speed between around $336 \mathrm{~m} \cdot \mathrm{s}^{-1}$ and $339 \mathrm{~m} \cdot \mathrm{s}^{-1}$. This exclusion is not as strong when the mountain wave field is included as figure 2b shows, and indeed the effective sound speed "follows" the ground as the air passes over the mountain (see, for instance, isoline $c=336 \mathrm{~m} \cdot \mathrm{s}^{-1}$ ). Nevertheless, it is clear that even in this case, the depth of the lower atmospheric duct substantially decreases as we move from the upstream side of the mountain to its top. This shrinking also manifests on the lee side, before that the flow reaches an abrupt expansion at around midslope to return to its upstream depth. Hence, for lower altitudes, these two effects produce a waveguide contraction as the flow passes over the mountain. Far above the mountain, the disturbances take the form of gravity waves that propagate upward. In the effective sound speed approximation framework, these gravity waves may be regarded as several acoustic waveguides in which relatively low-frequency acoustic waves can potentially propagate.

It is worthwhile noting that both the distorsion of the low level waveguide and the mountain wave field are not as intense for less stable situations (e.g. $J=0.5$, figure $2 \mathrm{~d}$ ). This is consistent with the fact that large values of $J$ favor downslope winds and Foehn. 
Comparison with the "mask" technique (figures 2c,d) demonstrates that for $J=0.5$ the lowest effective sound speed isoline follows the global curvature of the terrain, instead of being chopped by the mountain. As discussed in section IV, this effect helps infrasound signals to travel across the hill.

\section{Acoustic propagation in range-dependent media}

\section{A. Normal mode approach}

The approach follows the formulation of the initial-value problem adopted by Bertin et $a l .{ }^{6}$, among others, for range-dependent environments. Assuming that the modes couple adiabatically $^{24}$, the solution for the Fourier transform $\tilde{p}(z ; x, \omega)$ of the infrasound pressure fluctuations can be written as

$$
\tilde{p}(z ; x, \omega) \sim \sum_{j} \frac{a_{j}(\omega) \phi_{j}(z ; x, \omega)}{\sqrt{k_{j}(x, \omega)}} \mathrm{e}^{i \theta_{j}(x, \omega)}
$$

where $\phi_{j}, k_{j}, a_{j}$ and $\theta_{j}$ are respectively the $j$ th mode function, the corresponding modal wave number, amplitude and phase function. For a localized point-source at $x=z=0$ that emits a signal $s(t)$ we simply have $a_{j}(\omega)=\phi_{j}(0 ; 0, \omega)$, and the pressure fluctuation reads as

$$
p(z ; x, t)=\frac{1}{2 \pi} \int_{-\infty}^{\infty} \tilde{s}(\omega) \tilde{p}(z ; x, \omega) \mathrm{e}^{-i \omega t} \mathrm{~d} \omega
$$

where $\tilde{s}(\omega)$ is the Fourier transform of $s(t)$. For convenience, we denote the derivative of $\theta_{j}(x, \omega)$ by $k_{j}(x, \omega)$ and the frequency dependence is dropped for conciseness. Physically $k_{j}$ is the local (acoustic) wavenumber and the local phase speed is given by $c_{j}=\omega / k_{j}$. 
It is worth noting that the pressure fluctuation can generally be decomposed into propagating modes (along the $x$-axis direction) and evanescent modes, for which the imaginary part of $\theta_{j}$ is positive. Far downstream of the acoustic point-source, at a distance large compared to the wavelength, the evanescent modes are negligible and (10) is the correct expression to consider.

Substitution of (10) into the classical Helmholtz equation gives, to order unity,

$$
\frac{\partial^{2} \phi_{j}}{\partial z^{2}}+\left[\frac{\omega^{2}}{c^{2}}-k_{j}^{2}\right] \phi_{j}=0
$$

with the Neumann boundary condition expressing that the derivative of $\phi_{j}$ at $z=h(x)$ vanishes. For unbounded boundary layers, (12) must be supplemented by requiring a boundedness or outgoing-wave condition as $z \rightarrow \infty$. The solution of (12) then becomes $p \sim \mathrm{e}^{-\mu_{\infty} z}$ as $z \rightarrow \infty$, with the square-root function

$$
\mu_{\infty}(k)=\left[k^{2}-\frac{\omega^{2}}{c_{\infty}^{2}}\right]^{\frac{1}{2}}
$$

and $c_{\infty}$ denotes the effective sound speed in the limit $z \rightarrow \infty$. The function (13) depends on the variable $k$. Thus in the complex $k$-plane the branch cuts are to be inserted such that $-\pi / 2<\arg \left(\mu_{\infty}\right) \leq \pi / 2$. This choice of the branch cuts assures that as $z \rightarrow \infty$ the solution of (12) either goes to zero or represents an outgoing wave for all values of $k$ in the complex plane.

The branch cuts extend from the branch points $k=\omega / c_{\infty}$ and $k=-\omega / c_{\infty}$ to infinity in 
the complex $k$-plane. Over the initial region, far upstream of the mountain, the gravity wave field vanishes and we have $c_{\infty}=c_{0}+U_{0}$, whereas at ground-level the effective sound speed reaches its minimum $c(0,0)=c_{0}$. For a right-propagating wave the condition of trapping therefore imposes that the initial eigenvalues $k_{j}$ lie initially along the interval $c_{0}<\omega / k<$ $c_{0}+U_{0}$. As each mode propagates downstream both the vertical sound speed profile and the branch points $\omega / c_{\infty}(x)$ vary, and the local eigenvalues $k_{j}(x)$ slowly adapts to these changes. In this process, the phase velocity of some acoustic modes eventually becomes larger than $c_{\infty}$ and the associated trajectories terminate at a branch cut. Since it is not allowed to cross the branch cut it is therefore not possible to continue the eigensolution downstream of this point (and still satisfy the boundedness condition as $z \rightarrow \infty$ ). Therefore the corresponding modes are simply suppressed from the expansion (10).

In this work, the eigenfunctions and eigenvalues of (12) are calculated at a discrete set of ranges $x=x_{n}$ using the finite difference scheme used by Waxler et $a{ }^{48}{ }^{48}$, among others. The eigenvalues are obtained using a QR decomposition for $x=0$ and, for other ranges, the eigenvalues are tracked by using an iterative approach.

Since eigenfunctions are determined only up to a multiplicative constant, for definiteness we impose the normalization condition ${ }^{24}$

$$
\int_{h_{n}}^{\infty} \phi_{j}^{2}\left(x_{n}, z\right) \mathrm{d} z=1
$$



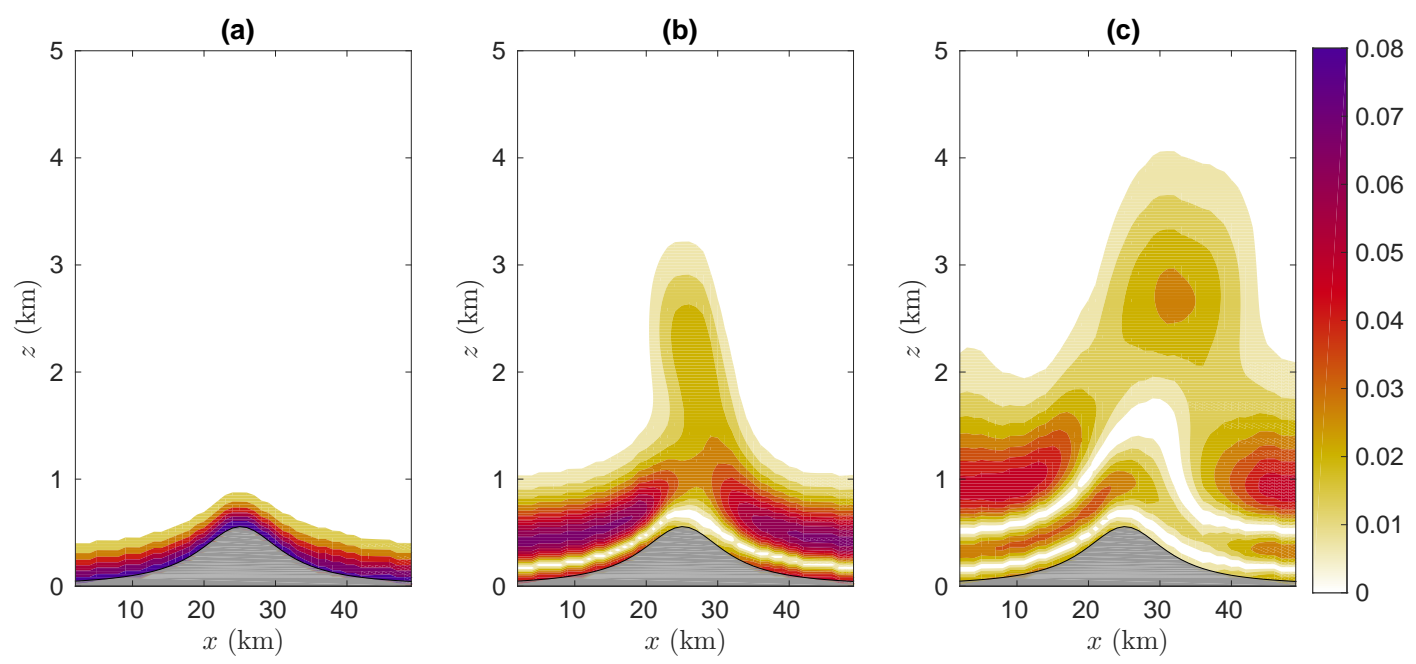

Figure 3: Eigenfunctions $\left|\phi_{j}\right|$ as functions of $x$ and $z$ for the first three modes (from left to right), and for a fixed frequency of $2.8125 \mathrm{~Hz}$. The background state is computed for $H_{N}=0.5$ and $J=1.5$ (cf. figure 2b). (a) $j=1$, (b) $j=2$ and (c) $j=3$.

where $h_{n}=h\left(x_{n}\right)$, together with the orthogonality condition

$$
\int_{h_{n}}^{\infty} \phi_{j}\left(x_{n}, z\right) \phi_{l}\left(x_{n+1}, z\right) \mathrm{d} z=\delta_{j l} .
$$

Anticipating the presence of upper level waveguides, the upper bound of integrals (14) and (15) was set to a sufficiently large value $z_{\text {top }}$ and the effective sound speed profile $c\left(x_{n}, z\right)$ was smoothly continued to higher altitudes when necessary. To assess the validity of the numerical results, it has been checked that the eigenvalues were not sensitive to changes in $z_{\text {top }}$, or to the choice of the continuation of $c\left(x_{n}, z\right)$ above $z_{\text {top }}$.

For illustrative purposes, figure 3 shows the eigenfunctions of the first three modes as $x$ increases along the source-receiver path, for a fixed frequency $\omega_{0}=2 \pi \times 2.8125 \mathrm{rad} . \mathrm{s}^{-1}$. For 
this frequency, these modes carry the dominant part of sound intensity over long distances and the expansion (10) can be truncated to $j \leq 3$, as discussed by Bertin et al. ${ }^{6}$. The first mode (figure 3a) is weakly sensitive to changes of the atmospheric flow as $x$ varies. The other two modes in figures 3b,c are clearly affected by the presence of gravity waves and indeed, ground-based attenuation is clearly visible in the vicinity of the ridge top elevation. Furthermore, figure 3c shows that the presence of mountain waves aloft allows the modes to be trapped in an upper duct. This result is discussed further in section IV.

\section{B. WKB treatment of the low-level waveguide}

To distinguish the effect due to the boundary layer shrinking from that due to the mountain wave at upper levels, and to gain insight onto the behavior of the trapped modes, we have obtained solutions to (12) using the following profile of effective sound speed

$$
\bar{c}(z)=\bar{c}_{0}+\bar{c}_{1} \tanh [z / \bar{\delta}],
$$

and the WKB approximation of (12). In (16) the parameters $\bar{c}_{0}, \bar{c}_{1}$ and $\bar{\delta}$ are chosen to minimize the integrated squared error between $c$ and $\bar{c}$ over the domain $h<z<z_{\max }$, where $z_{\max }$ is the depth of the low level waveguide, e.g. the lowest altitude such that

$$
\frac{d c}{d z}\left(z_{\max }\right)=0 .
$$

This definition ensures that the mountain wave is filtered out from the sound speed field and that the resulting waveguide width $z_{\max }$ varies slowly in the flow direction, as required by 
the classical asymptotic methods for modeling infrasound propagation ${ }^{24}$.

For fixed $x$, the filtered effective sound speed $\bar{c}$ in (16) is a strictly decreasing function of height and thus, $\omega^{2} / \bar{c}^{2}-k^{2}$ is a continuous function which involves a single turning point ${ }^{5}$ at $z=z_{0}(k)$. This choice allows us to use the Langer's formula ${ }^{26}$ to build the uniformly valid WKB approximation

$$
\bar{\phi}(z)=2 \sqrt{\pi} C\left[\frac{3}{2} \frac{S_{0}(z)}{\mu^{3}(z)}\right]^{\frac{1}{6}} \mathrm{Ai}\left\{\left[\frac{3}{2} S_{0}(z)\right]^{\frac{2}{3}}\right\}
$$

where Ai is the Airy function, and where the phase is given by

$$
S_{0}(z)=\int_{z_{0}}^{z} \mu(s) \mathrm{d} s
$$

and the turning point $z_{0}$ is the unique root of $\mu\left(z_{0}\right)=0$, where

$$
\mu(z)=\left[k^{2}-\frac{\omega^{2}}{\bar{c}^{2}(z)}\right]^{\frac{1}{2}}
$$

293 To ensure that the boundary condition at $z \rightarrow \infty$ is satisfied, the branch cuts are defined as for the function (13). Hence, using the leading asymptotic behavior of the Airy function for large $z$, (18) may be approximated by $\bar{\phi} \sim C \sqrt{\mu} \mathrm{e}^{-S_{0}}$, which is the leading order of the classical WKB approximation and the constant $C$ is determined by the normalization condition (14). It is important to point out at this time that this normalization condition plays a central role, especially when estimating the ground-based pressure $\bar{\phi}_{j} / \sqrt{k_{j}}$ as $x$ varies. In many cases, it can easily be verified that the closer to the ground the turning point is located, the greater the amplitude of the pressure field at ground level. 
Below the turning point, the path of integration must be deformed such that the squareroot function is continued into $\mu^{2}=-\mu^{2} \mathrm{e}^{i \pi}$ for $z<z_{0}$. On substituting this into (19), we note that $S_{0}^{2 / 3}$ is large and negative, and (18) can be simplified for $z \ll z_{0}$ by using the asymptotic behavior of the Airy function for negative argument ${ }^{1}$. To leading order, (18) may then be written as

$$
\bar{\phi}(z) \sim 2 C\left[-\mu^{2}(z)\right]^{-\frac{1}{4}} \cos \left\{\int_{z}^{z_{0}}\left[-\mu^{2}(s)\right]^{\frac{1}{2}} \mathrm{~d} s-\frac{\pi}{4}\right\}
$$

Now for $z=h$, on account of the (Neumann) boundary condition $\bar{\phi}_{z}(h)=0$, we obtain the constraint

$$
\int_{h}^{z_{0}(k)} \sqrt{-\mu^{2}(s)} \mathrm{d} s=\frac{\pi}{4}+j \pi,
$$

where $j$ is a nonnegative integer. Since $\mu^{2}$ depends on $k$, it appears that (22) determines the approximate value of $k_{j}$. In other words (22) defines the local dispersion relation where the streamwise station $x$ only appears as a parameter (which is not specified here for conciseness). As an additional bonus, equation (22) may be used to evaluate the effect of either downslope winds or mountain height on the local wavenumber. Upon totally differentiating the implicit function (22) for fixed $\omega$, and equating to zero, we obtain

$$
\frac{\mathrm{d} k}{\mathrm{~d} x}=-\frac{\int_{h}^{z_{0}} \frac{\omega^{2} \bar{c}_{x}}{\bar{c}^{3} \sqrt{-\mu^{2}}} \mathrm{~d} s+\frac{\mathrm{d} h}{\mathrm{~d} x} \sqrt{-\mu^{2}(h)}}{\int_{h}^{z_{0}} \frac{k}{\sqrt{-\mu^{2}}} \mathrm{~d} s},
$$

314 Where the terms of this ratio are the derivatives of (22) with respect to $k$ and $x$ and $\bar{c}_{x}$ is the 
Damiens, Millet and Lott, J. Acoust. Soc. Am., p. 22

derivative of $\bar{c}$ with respect to $x$. Similarly, we use the notations $k_{x}$ and $h_{x}$ for the derivatives of $k$ and $h$ with respect to $x$, respectively. Application of (23) for $\bar{c}_{x}>0$ and $h_{x}>0$ leads to $k_{x}<0$. This means that the phase speed $\omega / k$ increases as the flow speeds up or when $h$ increases.

\section{Impact of mountain waves on the normal modes}

In order to obtain the pressure signal from (10), the modal wave numbers $k_{j}$, or equivalently the phase velocities $c_{j}=\omega / k_{j}$ are required. For range-dependent environments, these quantities are obtained as functions of $\omega$ and $x$ either by solving (12) numerically or by using the WKB approximation, as described in section III.B. Figure 4 show contours of the phase velocity in the $(\omega, x)$-plane for the first three modes $[j \leq 3$ in (10)] and the two effective sound speed fields considered in figures $2 \mathrm{a}$ and $2 \mathrm{~b}$. In figures $4 \mathrm{a}, \mathrm{b}, \mathrm{c}$ the numerically obtained results are represented in colors, when the mountain "mask" is applied, and the black curves give the corresponding WKB values. Figures 4d,e,f show the results obtained when the mountain flow dynamics is considered. Two important curves are also plotted as red and blue curves. These curves are obtained for each eigenvalue by decreasing $\omega$, the location $x$ being fixed. Starting from an initial value, the phase velocity $c_{j}$ increases up to the maximum sound speed $c_{\max }$ as $\omega$ decreases. This behavior allows to identify the so-called cut-off frequency of the low-level waveguide, for which we have $c_{j}(x, \omega)=c\left(x, z_{\max }\right)$, and which is referred to as $\omega_{j}^{+}(x)$ in the following (blue curve). For $\omega=\omega^{+}$the eigenvalue obtained 

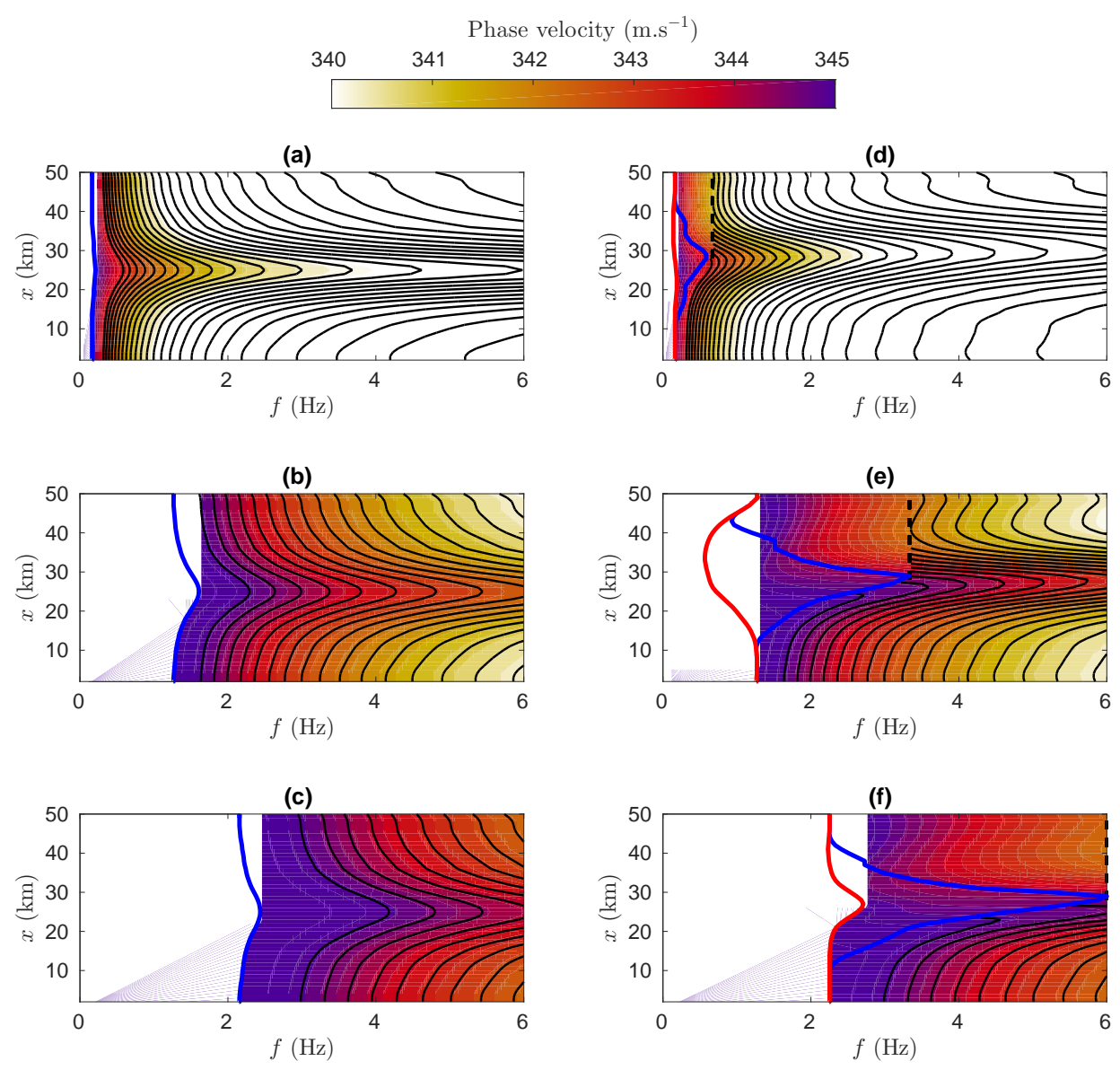

Figure 4: Phase velocity $c_{j}$ for the first three modes (from top to bottom) as a function of streamwise location and frequency, for $H_{N}=0.5$ and $J=1.5$. (a,d) $j=1$; (b,e) $j=2$; (c,f) $j=3$. The results obtained by applying a "mask" onto the effective sound speed field are given on the left (a-c). The figures on the right (d-f) show the impact of mountain wave dynamics. Black, red and blue curves give the WKB prediction and the cut-off frequencies $\omega^{-}$and $\omega^{+}$as defined in section IV. 
from (22) crosses a branch cut of (13) and thus, the WKB approximation fails to give a result for $\omega<\omega^{+}(x)$. Physically this condition may be interpreted as the requirement that the mode is not to be trapped in the low level duct. On the other hand the eigenvalue can be computed directly from (12) for lower frequencies, so that the eigenvalue reaches a terminal value for which we have $c_{j}(x, \omega)=c_{\max }(x)$. This value is referred to as $\omega_{j}^{-}(x)$ (red curve). It turns out that $\omega^{-}$is not defined when considering the "mask" effect alone (figures $\left.4 a, b, c\right)$, essentially because in this case we have a single waveguide. Therefore, when mountain waves are present (figures $4 \mathrm{~d}, \mathrm{e}, \mathrm{f}$ ), the region $\omega_{j}^{-}<\omega<\omega_{j}^{+}$corresponds to frequencies for which the eigenfunction $\phi_{j}$ penetrates up to the moutain wave field and can be confined within an upper level waveguide, as depicted in figure 3c for $x$ lying in the range $25-35 \mathrm{~km}$. This is an indication that at sufficiently low frequencies strong interaction between modes and mountain waves may occur.

As detailed in section III.A, a mode is not allowed to cross the branch cut, a situation that occurs for $\omega<\omega^{-}(x)$. The basic problem here is that as soon as the phase velocity of the locally wave solution becomes larger than the maximum effective sound speed, it is not possible to find a solution that remains bounded in the limit $z \rightarrow \infty$. Within the framework of slowly varying media, this condition translates into $a_{j}(\omega)=0$ for $\omega<\omega^{-}(0)$. Physically this condition may be interpreted as the requirement that the mode does not propagate along the source-receiver path, for $x>0$. The corresponding regions in the $(\omega, x)$ plane are 
represented by blank areas in figures 4d,e.

For fixed but quite high frequencies (greater than $1 \mathrm{~Hz}$ typically), the phase velocity of the first mode, which is also the slowest mode (figure 4a,d), increases as we approach the ridge before decreasing in the lee side. Although this effect is less strong in presence of moutain flow, this behavior can be captured qualitatively using the "mask" technique and the WKB approximation. In fact this mode, which is confined in the vicinity of the ground, find its way through the ridge, even when the waveguide is substantially shrinked by mountain wave dynamics (figure $4 \mathrm{~d}$ ) or chopped by the ridge (figure $4 \mathrm{a}$ ). The excellent agreement with the WKB approximation suggests that the mode essentially adjusts to the vertical shrinking of the waveguide, the increasing in its phase velocity being correctly predicted by equation (23) with $\bar{c}_{x}=0$. For lower frequencies (less than $1 \mathrm{~Hz}$ ), the discrepancies between the results obtained with the "mask" technique and the mountain flow are more pronounced, and essentially occur in the region where the low level duct fails to trap the modes, e.g. when lines of constant phase velocity intersect the blue curve. This is detailed in the following for the next two modes. For $j=1$, we observe an overall agreement between the results obtained by solving (12) numerically or by using the WKB approximation. From a practical standpoint, this demonstrates that the interaction between infrasound and mountain flows can adequately be predicted at a low numerical cost, through finding the first maximum in the local effective sound celerity and using the WKB approximation. This approach, 
however, is justified only if we can neglect the contribution of other modes $(j>1)$.

In computing the phase velocity for the other modes $(j>1)$, we observe that the mask technique fails in predicting important changes. Primarily, figures $4 \mathrm{~b}, \mathrm{c}$ show that the cut-off frequency $\omega^{+}$substantially increases as we approach the ridge top, and reaches its maximum at $x_{0}=25 \mathrm{~km}$. As discussed in section III.A, when the condition $\omega<\omega^{+}\left(x_{0}\right)$ is satisfied downstream $x_{0}$, the mode is simply suppressed. In presence of mountain waves (figures $4 \mathrm{e}, \mathrm{f})$ the low level waveguide is extremely shrinked, and the penetration of $\omega^{+}(x)$ into the $(\omega, x)$-plane is very pronounced. This effect is essentially due to Foehn, which shifts the maximum cutt-off frequency $\omega^{+}(x)$ on the leeward side of the ridge, at a distance of approximately $30 \mathrm{~km}$ (figure 4e,f). Hence, immediately downstream this location, the mode obtained with the one-turning-point WKB approximation (i.e. when mountain waves are filtered out) must be suppressed, as shown in figures 4e,f. This is not the case when mountain waves are considered and indeed, the fact that the mode remains propagating in the horizontal direction for $x>x_{0}$ is essentially due to the emergence of multiple possible upper ducts above the mountain. For $j=2$, we even see that the cut-off frequency of the upper duct $\omega^{-}$decreases as we pass over the ridge and thus, the contribution of the mode has to be maintained in (10). This finding is in strong contrast with that obtained using the "mask" technique. On the other hand, and for $j=3$, figure $4 \mathrm{f}$ shows that $\omega^{-}$increases as we move closer to the ridge. This means that the mountain wave pattern failed in ducting the 
mode that escapes from the low-level duct. Finally it is important to notice that for smaller values of $J$ ( $H_{N}$ being constant), the boundary layer tends to follows the global curvature of the terrain, thereby yielding a significant number of modes to travel over the mountain, whereas the upper bound $\omega^{+}$obtained with the "mask" technique penetrates much more into the $(\omega, x)$-plane.

Figure 5 shows the sound intensity $\left|\phi_{j} / \sqrt{k_{j}}\right|$ at ground level $z=h(x)$, as a function of $x$ and $\omega$, for the first three modes. The magnitude of the contours are labelled in decibel, with a reference sound intensity computed at $x=0$. Results are given for the two effective sound speed profiles defined above and depicted in figures $2 \mathrm{a}, \mathrm{b}$. The contours in color are for the results obtained by solving numerically (12), and the black curves give the one-turning-point WKB approximation. Red curves and blue curves represent the cut-off frequencies $\omega_{j}^{-}$and $\omega_{j}^{+}$, as in figure 4. Figures 5d,e,f, essentially show strong attenuation in the region $\omega_{j}^{-}<\omega<$ $\omega_{j}^{+}$. These attenuations are due to strong interactions between the acoustic waves and the mountain waves, the latter creating new acoustic waveguides at higher altitudes, as discussed previously. The energy leaks that follow the tunneling effect for sound waves (cf. figure 3b,c) and the standard requirement that the integral of $\phi_{j}^{2}$ is fixed to one [condition (14)], lead to strong attenuations at ground level. These attenuations are more pronounced for higher indices, simply because the corresponding turning points, at $x=0$, are closer to $z$ max. Since the phase velocity adapts to the local environment encountered by the sound wave, 


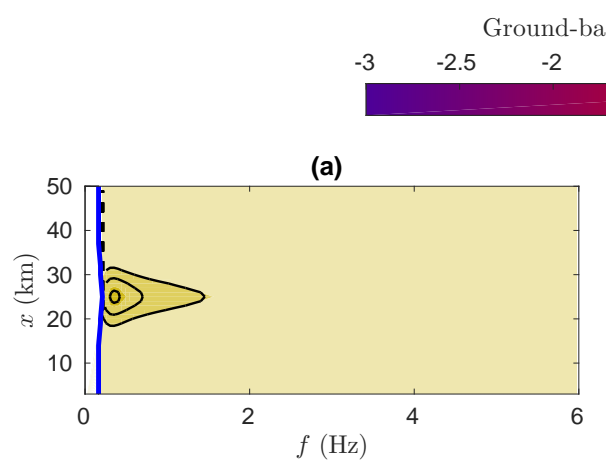

(b)
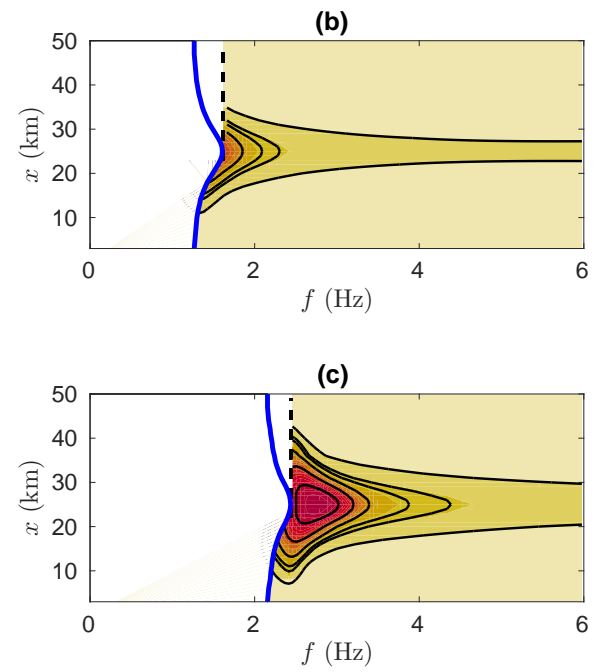

(d)

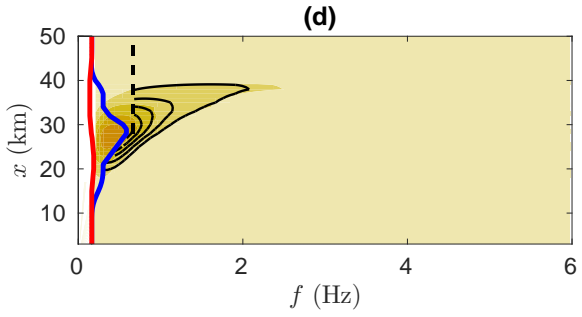

(e)
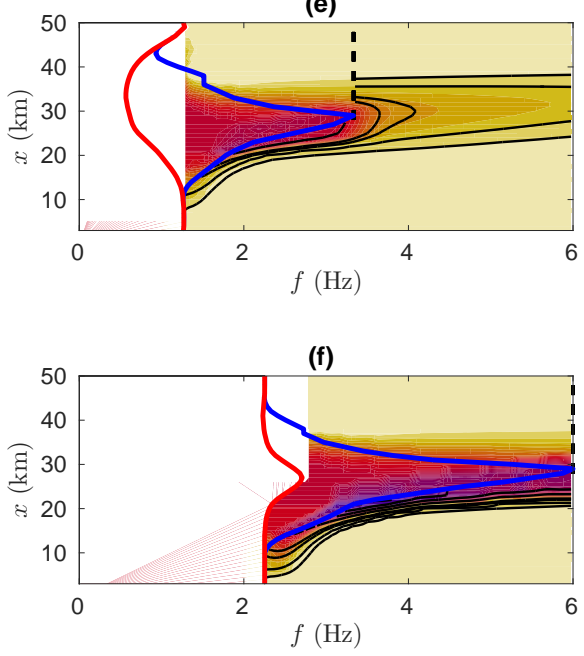

Figure 5: Ground-based sound intensity $\left|\phi_{j} / \sqrt{k_{j}}\right|$ for the first three modes as a function of streamwise location and frequency, for $H_{N}=0.5$ and $J=1.5$. (a,d) $j=1$; (b,e) $j=2$; $(\mathrm{c}, \mathrm{f}) j=3$. The WKB prediction is superimposed in black contours. Red and blue curves correspond of those of figure 4 .

the highest modes are more likely to leave the low-level waveguide.

While the ground-based attenuation of sound intensity can be qualitatively understood when the mode shifts to upper-level waveguides (for $\omega_{j}^{-}<\omega<\omega_{j}^{+}$), it is less clear why 
it occurs when the waveguide shrinks, as figures $5 \mathrm{a}, \mathrm{b}, \mathrm{c}$ show for $\omega>\omega^{+}$. This behavior appears to contradict the normalization requirement, which a priori results in surface amplifications rather than surface attenuations. However, using the WKB approximation, the apparent contradiction is resolved by the recognition that the proper measure of the size of the dispersive region, $z_{0}-h$ is always smaller than its value at $x=0$. Based on the above discussions, clearly the surface attenuation is a combination of the emergence of upper-level waveguides as well as depth reduction of the low-level waveguide.

\section{Impact of mountain waves on signals}

In the previous section we have seen how the normal modes, in which the structure over the whole $(\omega, x)$-plane can be delineated, are attenuated by mountain waves, and we have given a general condition by which this interaction can be characterized, in terms of the cut-off frequencies $\omega^{-}$and $\omega^{+}$. To measure the extent to which these effects are significant when the sources of infrasound are localized in both space and time, we next calculate ground-based waveforms, using the FFT algorithm ${ }^{7}$. A source function is introduced in the form

$$
s(t)=K \mathrm{e}^{-\frac{t-T_{0}}{\sigma^{2}}} \cos \left(2 \pi f_{c} t\right),
$$

where $T_{0}=10 \mathrm{~s}, f_{c}=3 \mathrm{~Hz}$ and $\sigma=1 / 5$. The parameters are adjusted such that the maximum frequency is $6 \mathrm{~Hz}$, with a leading frequency of $3 \mathrm{~Hz} . K$ is a suitable coefficient that yields a normalized pulse. This source transfers most of its energy onto the first three 
Damiens, Millet and Lott, J. Acoust. Soc. Am., p. 30

431

modes $[j \leq 3$ in (10)] which are the modes of greatest contribution when the frequency is relatively low. For this reason, the modal expansion is truncated to these modes in the following.

The normalization of signals obtained for different locations downstream the mountain is fixed so that the amplitudes can be compared to each other. The global effect of the mountain can be summarized by means of the attenuation

$$
1-\frac{I(x)}{I_{0}(x)},
$$

where the sound intensity at ground level $(z=h)$ is defined as

$$
I(x)=\int_{0}^{\infty}[p(h ; x, t)]^{2} \mathrm{~d} t
$$

where the waveform $p(h ; x ; t)$ is obtained from (11) and (10), with $j \leq 3$, and by solving the Helmholtz equation (12) numerically. Here $I_{0}$ is a reference sound intensity obtained by taking $x=0$ in (26). This choice allows the results to be compared with the classical mountain-free range-independent case $\left(i . e\right.$. when the effective sound speed is given by $c_{0}+$ $U(z))$, which is used in the infrasound research community.

In this section, we proceed systematically and vary the Richardson number between 0.25 and 2 and the non-dimensional mountain height $H_{N}$ between 0.2 and 0.8 . As discussed in section IV, the sound speed modifications are intimately linked to the mountain flow situations. To measure the downslope wind amplitude and Foehn, and following Lott ${ }^{29}$, we 
use

$$
A=\max _{\substack{2 z<H_{N} \\ 0<x<2 F}}\left[\frac{u^{\prime}(x, z)}{U(H)}\right],
$$

which is the maximum of the ratio between the horizontal wind disturbance along the foothill and the background wind at the top of the hill. Typically, when $A$ approaches and exceeds 1 , the dynamics induces wind amplitudes that are either equal to or exceed the amplitude of the winds at the summit of the ridge. In other words, the flow speeds up along the ridge, and this occurs easily for $J>1$.

Before proceeding systematically we present here three cases that illustrate the general results that will conclude the paper. Case $\mathrm{I}$ is defined by $\delta=600 \mathrm{~m}$ and $H=250 \mathrm{~m}$, which corresponds to a relatively small mountain and a pronounced shear. In terms of dimensionless parameters, we have $J=0.75$ and $H_{N}=0.3$ so that the downslope wind amplitude is $A=0.75$. Case II is associated with a larger depth $\delta=1 \mathrm{~km}$ and a higher mountain $H=600 \mathrm{~m}$. The corresponding dimensionless parameters are given by $J=2$ and $H_{N}=0.8$ so that $A=3$, which reflects intense downslope winds. In order to estimate the role of stability, we keep $H_{N}=0.8$ and consider a much less stable flow with a Richardson number $J=0.3$, as a third Case III. This last case corresponds to a situation for which we have $H / \delta=H_{N} / \sqrt{J} \simeq 1.5$ and thus, we can expect that most of the modes are obstructed by the mountain when the "mask" technique is used.

The resulting acoustic signals associated with cases I, II and III are shown in figure 6 
(a)

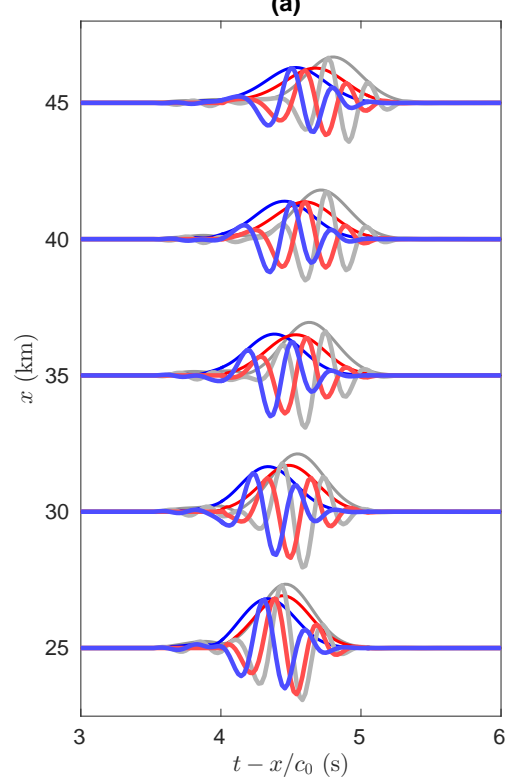

(b)

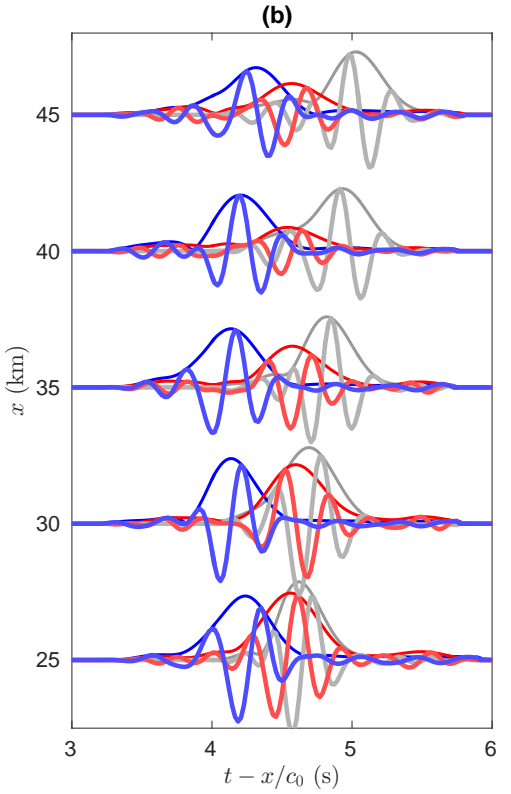

(c)

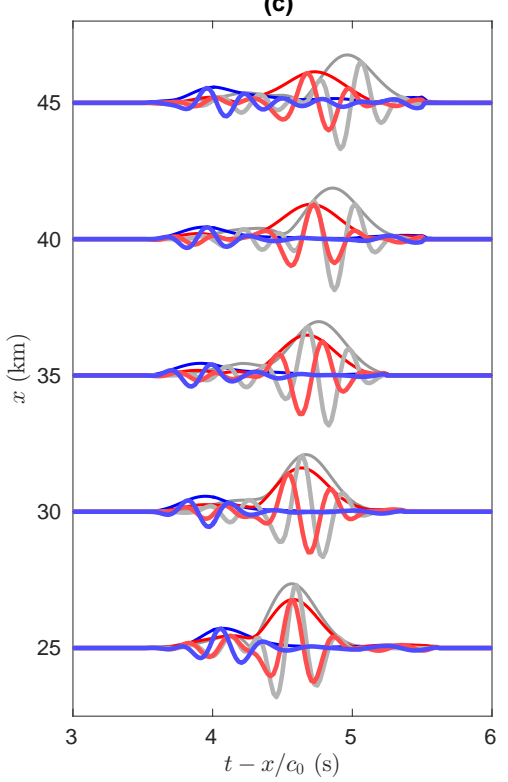

Figure 6: Waveforms obtained for cases I (a), II (b) and III (c) as functions of the retarded time $t-x / c_{0}$ without (blue) and with (red) interaction between the mountain and the boundary layer. The signals obtained for an unperturbed range-independent case (without mountain and mask) are plotted in gray, for reference. Case I: $J=0.75$ and $H_{N}=0.3$; case II: $J=2$ and $H_{N}=0.8$; case III: $J=0.3$ and $H_{N}=0.8$. The source is defined by (24). for different locations downstream the mountain. The blue and red colors correspond to waveforms computed by applying the "mask" technique and by solving mountain flow dynamics, respectively. Waveforms obtained for the unperturbed range-independent profile $c_{0}+U(z)$ are plotted in gray. The envelope of signals is plotted in thinner line, using the Hilbert transform. Figure 6 shows evidence that the interaction between the mountain flow 
Damiens, Millet and Lott, J. Acoust. Soc. Am., p. 33

and the acoustic wave may give rise to attenuation or amplification of ground-based signals, depending on the Richardson number. While the impact of the mountain on the groundbased signals is moderate for Case I (figure 6a), for which the attenuation does not exceed $13 \%$, Case II (figure 6b) gives rise to attenuations as large as $48 \%$ at $x=40 \mathrm{~km}$. This attenuation is mainly due to the fact that the first mode is no longer trapped in the low level waveguide and thus, a large part of the energy is lost at higher altitudes through interactions with mountain waves. On the other hand, case III (figure 6c) shows that the mountain wave dynamics may favor the passage of acoustic waves, mitigating the "mask" effect. The essential constrast with Case II is that, despite a strong reduction of its height, the incoming waveguide slips over the mountain rather than being destroyed over the winward side. The acoustic path then follows the global curvature of the terrain and the sound intensity is $80 \%$ larger than that obtained with the "mask" technique.

As discussed above, the signals obtained for the three cases considered in figure 6 do not cover all situations. In order to estimate how the mountain wave dynamics impacts the infrasound measurement, the ground-based attenuations (25) are first computed as functions of $x$, and then averaged over two intervals $x_{0}<x<x_{1}$ and $x_{1}<x<x_{2}$, with $x_{0}=25 \mathrm{~km}$, $x_{1}=40 \mathrm{~km}$ and $x_{2}=50 \mathrm{~km}$. The process is repeated for different values of $J$ and $H_{N}$ so as to obtain a complete portrait of averaged attenuations in time domain. Figures 7 and 8 show typical results for the first and second intervals, respectively. The first interval 
(a)

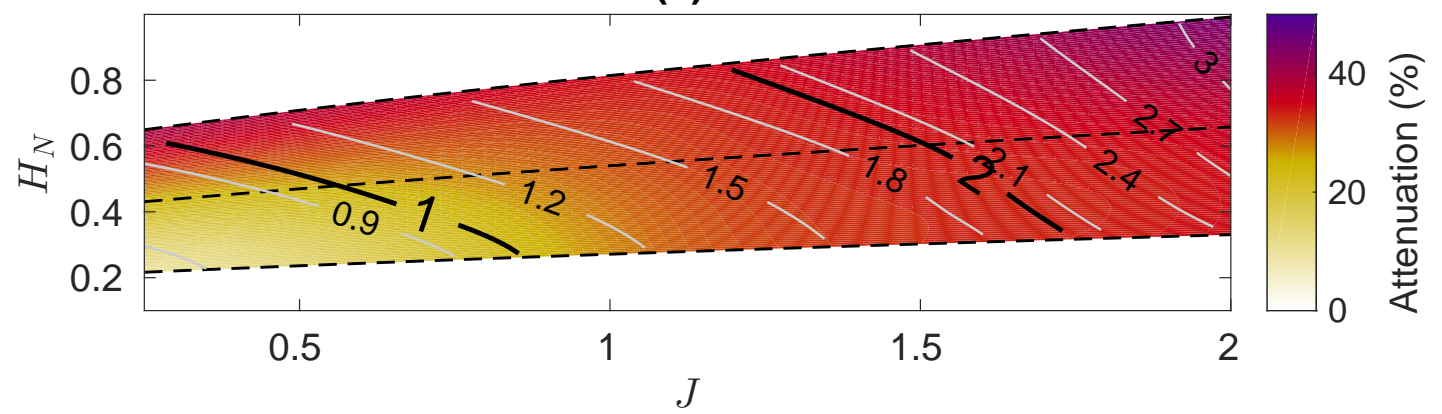

(b)

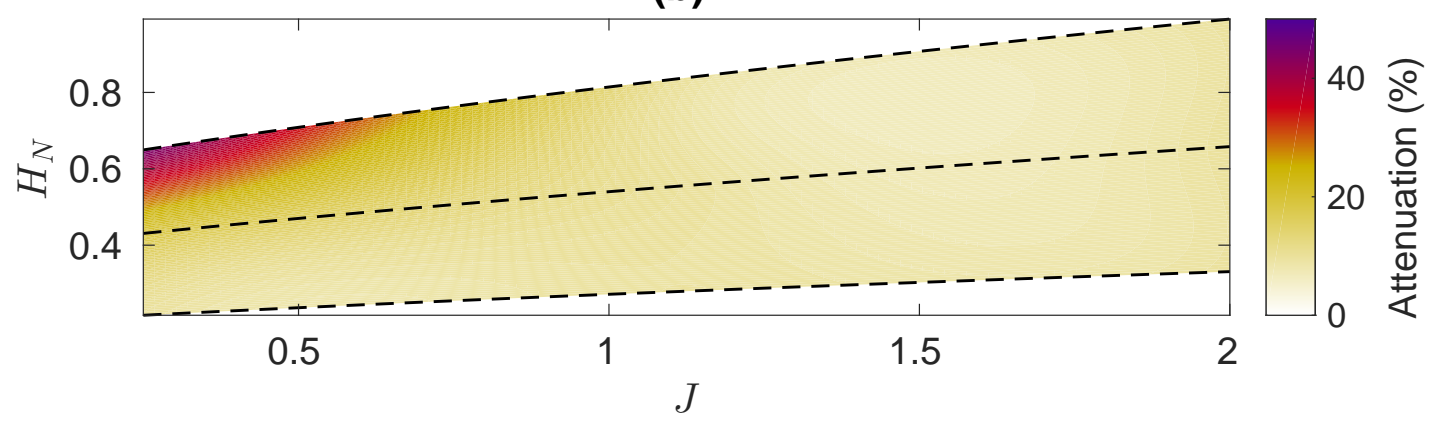

Figure 7: Far-field averaged attenuation downstream the mountain, in the range $25-40 \mathrm{~km}$ as a function of $J$ and $H_{N}$ with mountain wave disturbances (a) and the "mask" effect alone (b). The downslope wind amplitude $A$ is given by black and white contours.

${ }_{489} x_{0}<x<x_{1}$ is adopted here to quantify the infrasound attenuation on the leeward flank 490 of the ridge. Firstly, figure 7a shows that the mountain flow produces larger attenuations 491 than that obtained with the "mask" technique (in figure 7b). Furthermore, even though the shrinking of the waveguide by the Foehn produces strong attenuations ( $A$ is almost everywhere larger than 1), a significant fraction of the attenuation is indeed associated 
with sound propagation within upper level waveguides, through local adjustments of few normal modes, as discussed in length in section IV. This is typically the case for relatively large $J(J>1.5)$ and small $H_{N}$, in the range $0.2<H_{N}<0.4$. In this region the sound intensity on the lee-side flanck of the ridge is attenuated by a factor of $30 \%$ (figure $7 \mathrm{a}$ ) and decreases down to about $20 \%$ far downstream (figure 8a). Secondly, comparisons of figures $7 \mathrm{a}$ and $7 \mathrm{~b}$ show that at low Richardson numbers $(J<0.5)$ and for high mountains $\left(0.6<H_{N}<0.7\right)$ attenuation is mainly due to the "mask" effect, which produces a strong reduction of the waveguide height. The second interval is used to capture the far-field sound attenuation downstream the mountain without including the constructive/destructive interference effects associated with local changes of phases. In fact, at about two or three mountain half-widths downstream of the maximum height location, the modes recover their initial characteristics for $x \rightarrow \infty$, unless they reach a branch cut as discussed in section II.A. Owing to these changes in the resulting modal expansion (10), a residual attenuation is expected far downstream the mountain. This attenuation is irreversible in the sense that the full set of eigenvalues at $x=0$ is not recovered downstream the mountain. Comparison of figures $7 \mathrm{a}$ and $8 \mathrm{a}$ shows that this effect is apparent at relatively high Richardson numbers, in the top right corner of figure 7a. Finally, it is important to point out that another striking result here is that for large values of $H_{N}$ and narrow waveguides (small $J$ ), the mountain flow dynamics favor infrasound propagation, as discussed in section IV. 
(a)

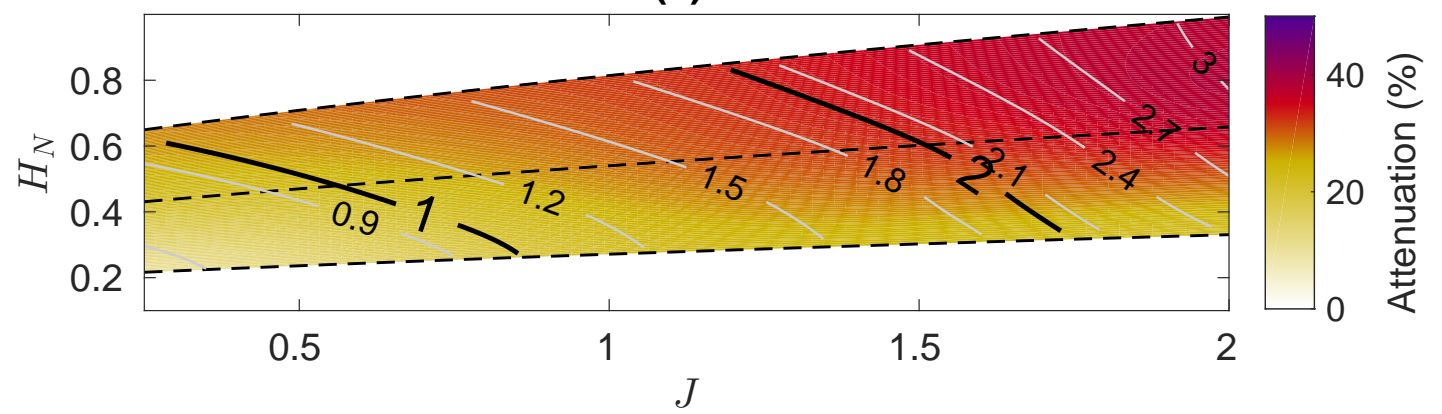

(b)

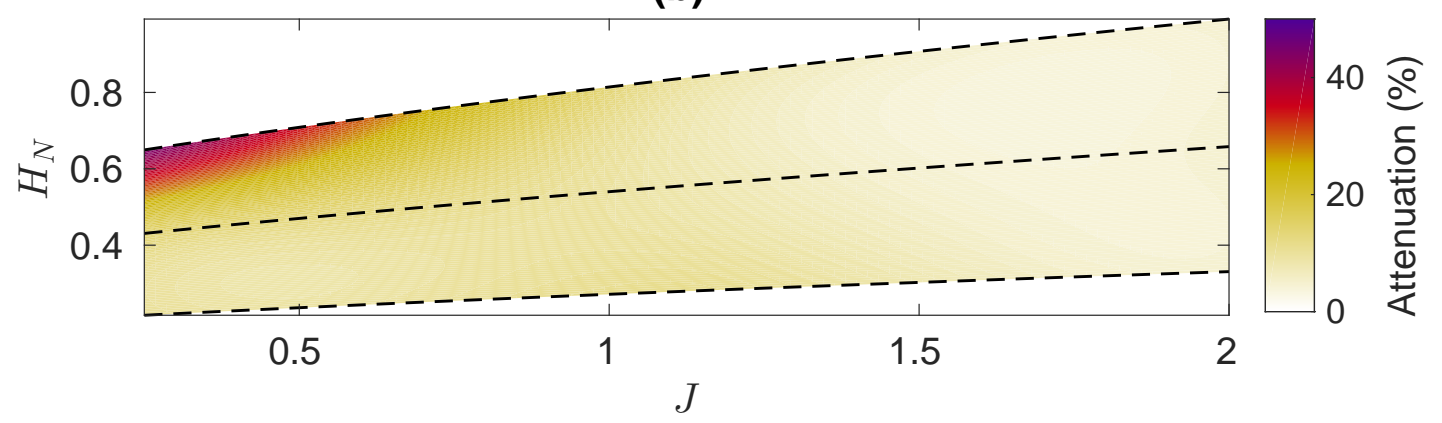

Figure 8: Far-field averaged attenuation, in the range $40-50 \mathrm{~km}$ as a function of $J$ and $H_{N}$ with mountain wave disturbances (a) and the "mask" effect alone (b). The downslope wind amplitude $A$ is given by black and white contours.

\section{Conclusions}

In this paper, we have examined the propagation of sound within mountain flows.

The mountain flow model is based on the integration of the linear inviscid Taylor-Goldstein equation, forced by a nonlinear surface boundary condition. To calculate infrasound signals, 
we also used a range-dependent normal mode approach, which allows the decomposition of the acoustic pressure field into distinct normal modes. The basic assumption introduced in the present work is that the acoustic modes couple adiabatically, $i$. e. without any transfer of energy to higher or lower modes. Ground-based signals were computed using Fourier synthesis of frequency-domain solutions, for a given ground-based broadband acoustic source.

The central result of this paper is that mountain wave dynamics may lead to strong attenuation or amplification of upcoming acoustic waves, regarding to the direct "mask" effect the mountain has on acoustic propagation. For a stable flow $(J \geq 1)$ the mountain wave dynamics produces large horizontal winds and buoyancy disturbances at low level that result in intense downslope winds and Foehn. When the downslope wind is less intense $(J<1)$, the flows can reinforce the acoustic waveguide over the mountain and lead to a signal of greater amplitude compared to that obtained by the "mask" effect. The acoustic waveguide is then strongly impacted which leads to a new kind of acoustic (reversible) absorption that can be related to local adjustments of few normal modes. It is worthwhile to point out here that acoustic absorption is mainly governed by the Richardson number, and more precisely by the critical value $J \simeq 1$, which is also a transition regime for mountain wave dynamics.

In striking contrast to this local behavior of acoustic modes is the sound attenuation far downstream from the mountain. This second type of absorption is due to irreversible 
Damiens, Millet and Lott, J. Acoust. Soc. Am., p. 38

536

537

538

539

processes that are intimately connected to leaking modes along the source-receiver path. While leaking modes are known to play a role in the transient waveform, the classical practice is to neglect the contributions from these modes at large horizontal distances from explosions in the atmosphere. This approach, however, ignores range-dependence of the environment. These modes may be "activated" by a point-source in the form of classical waveguide modes and then decay exponentially with increasing distances far downstream from the mountain, as a result of the atmospheric state evolution. In this way, the corresponding component involving these modes vanishes far downstream from the mountain. This results in absorption farther downstream from the mountain, even though the background state recovers its initial state (e.g. upstream from the mountain).

The present work presents our current understanding of acoustic absorption due to mountain wave dynamics with emphasis upon a modal description of the acoustic field, including static stability effects. Though the present analysis does not answer all the questions regarding the complex phenomenon of absorption, it has shown how a range-dependent analysis can provide some insight into the interaction of acoustic waves and mountain wave fields. Other aspects that may give rise to additional dissipation have not been fully explored, such as, interaction of infrasound waves with ground and turbulence. However, the mountain wave model used in this study cannot predict the turbulence associated with GW breaking, a process that occurs for small $J$. While a rough estimate of the complex impedance effect 

573 climate $^{28}$. dominates.

gives an absorption of $1 \%$ for the cases considered in this study, the role of turbulence is more complex to quantify. The main difficulty is that the adiabatic approximation ceases to apply when the turbulence correlation length and acoustic wavelength are of the same order of magnitude. Some preliminary calculations have been made by the authors for estimating far-field absorption, using the techniques described in this paper, but with mode couplings and complex imaginary part of the grounding impedance. The results show good agreement with that obtained in the present work, except for cases where fine-grained turbulence

The present work is also related to the more general issue of incorporating unresolved GW variability in infrasound propagation calculations. Recent works ${ }^{25 ; 21 ; 14}$ suggest that the mismatch between simulated and observed signals is related to the fact that the atmospheric specifications used in most studies do not adequately represent internal gravity waves. In the Atmospheric General Circulation Models which are used to produce the atmospheric specifications, these GWs are represented by parameterizations and in return, these parameterizations can be used to predict the GWs field used in infrasound studies. This is the approach followed by Drob et al. ${ }^{14}$, in which the global spectral scheme of Hines ${ }^{22}$ is used to estimate the effect of GWs on infrasound time arrivals. The interesting aspect of using the model proposed by Hines ${ }^{22}$ is that it allows obtaining GW fields that give rise to the right 
Damiens, Millet and Lott, J. Acoust. Soc. Am., p. 40

\section{REFERENCES}

1. M. Abramowitz, and I. A. Stegun, Handbook of mathematical functions: with formulas, graphs, and mathematical tables, Vol. 55. National Bureau of Standards Applied Mathematics Series (1964), p. 1046.

2. J. D. Assink, G. Averbuch, P. S. M. Smets, and L. G. Evers, "On the infrasound detected from the 2013 and 2016 DPRK's underground nuclear tests", Geophys. Res. Lett., 43, 3526-3533 (2016).

3. J. D. Assink, R. Waxler, and D. Drob, "On the sensitivity of infrasonic traveltimes in the equatorial region to the atmospheric tides", J. Geophys. Res. Atmos., 117, D01110 (2012).

4. K. Attenborough, K. M. Li, and K. Horoshenkov, "Predicting outdoor sound", CRC Press (2006), p. 485.

5. C. M. Bender, and S. A. Orszag, Advanced mathematical methods for scientists and engineers I: Asymptotic methods and perturbation theory, Springer Science and Business Media (2013), p. 593.

6. M. Bertin, C. Millet, and D. Bouche, "A low-order reduced model for the long-range 
Damiens, Millet and Lott, J. Acoust. Soc. Am., p. 41 propagation of infrasounds in the atmosphere", J. Acoust. Soc. Am., 136(1), 37-52 (2014).

7. E. O. Brigham, and R. E. Morrow, "The fast Fourier transform", IEEE spectrum, 4(12), 63-70 (1967).

8. J. Candelier, S. Le Dizès, and C. Millet, "Inviscid instability of a stably stratified compressible boundary layer on an inclined surface", J. Fluid Mech., 694, 524-539 (2012).

9. W. Cheng, F., M. B. Parlange, and W. Brutsaert, "Pathology of Monin-Obukhov similarity in the stable boundary layer", J. Geophys. Res., 110, D06101 (2005).

10. G. Chimonas, and C. J. Nappo, "Wave drag in the planetary boundary layer over complex terrain", Boundary-layer meteorology, 47, 217-232 (1989).

11. I. P. Chunchuzov, G. A. Bush, and S. N. Kulichkov, "On acoustical impulse propagation in a moving inhomogeneous atmospheric layer", J. Acoust. Soc. Am., 88(1), 455-461 (1990).

12. I. Chunchuzov, S. Kulichkov, A. Otrezov, and V. Perepelkin, "Acoustic pulse propagation through a fluctuating stably stratified atmospheric boundary layer", J. Acoust. Soc. Am., 117(4), 1868-1879 (2005). 
Damiens, Millet and Lott, J. Acoust. Soc. Am., p. 42

13. F. Damiens, F. Lott, C. Millet, and R. Plougonven, "An adiabatic Foehn mechanism", Under consideration for publication in Quaterly J. of the Royal Meteorol. Soc. (2017).

14. D. P. Drob, D. Broutman, M. A. Hedlin, N. W. Winslow, and R. G. Gibson, "A method for specifying atmospheric gravity wavefields for long-range infrasound propagation calculations", J. Geophys. Res. Atmos., 118(10), 3933-3943 (2013).

15. D. R. Durran, "Mountain waves and downslope winds". AMS Meteorological Monographs, 23, 59-83 (1990).

16. J. A. Dutton, Dynamics of atmospheric motion, Dover Publications, Inc., New-York (1995), p. 617.

17. D. Fee, R. Waxler, J. Assink, Y. Gitterman, J. Given, J. Coyne, P. Mialle, M. Garces, D. Drob, D. Kleinert, and R. Hofstetter, "Overview of the 2009 and 2011 Sayarim infrasound calibration experiments", J. Geophys. Res. Atmos., 118(12), 6122-6143 (2013).

18. D. C. Fritts, and M. J. Alexander, "Gravity wave dynamics and effects in the middle atmosphere", Rev. Geophysics, 41(1), 1003 (2003).

19. O. A. Godin, "An effective quiescent medium for sound propagating through an inhomogeneous moving fluid", J. Acous. Soc. Am., 112, 1269-1275 (2002).

20. E. E. Gossard, and W. H. Hooke, "Waves in the atmosphere: atmospheric infrasound 
Damiens, Millet and Lott, J. Acoust. Soc. Am., p. 43 and gravity waves-their generation and propagation". Elsevier Scientific Publishing Company (1975), p. 456.

21. M. A. Hedlin, and D. P. Drob, "Statistical characterization of atmospheric gravity waves by seismoacoustic observations", J. Geophys. Res. Atmos., 119(9), 5345-5363 (2014).

22. C. O. Hines, "Doppler-spread parameterization of gravity-wave momentum deposition in the middle atmosphere. Part 2: Broad and quasi monochromatic spectra, and implementation", J. Atmos. Terr. Phys., 59(4), 387-400 (1997).

23. L. N. Howard, "Note on a paper of John W. Miles", J. Fluid Mech., 10(04), 509-512 (1961).

24. F. B. Jensen, W. A. Kuperman, M. B. Porter, and H. Schmidt, Computational ocean acoustics, Springer Science and Business Media (2011), p. 794.

25. J. M. Lalande, and R. Waxler, "The interaction between infrasonic waves and gravity wave perturbations: Application to observations using UTTR Rocket Motor Fuel Elimination Events", J. Geophys. Res. Atmos., 121(10), 5585-5600 (2016).

26. R. E. Langer, "The asymptotic solutions of ordinary linear differential equations of 
Damiens, Millet and Lott, J. Acoust. Soc. Am., p. 44

the second order, with special reference to a turning point", Trans. Amer. Math. Soc., 67(2), 461-490 (1949).

27. R. R. Long, "Some aspects of the flow of stratified fluids; 1. A theoretical investigation", Tellus, 5, 42-58 (1953).

28. F. Lott, and C. Millet, "The representation of gravity waves in atmospheric general circulation models (GCMs)", In Infrasound monitoring for atmospheric studies, Springer Netherlands (2010), pp. 685-699.

29. F. Lott, "A new theory for downslope windstorms and trapped mountain waves", J. Atmos. Sci., 73(9), 3585-3597 (2016).

30. M. H. McKenna, R. G. Gibson, B. E. Walker, J. McKenna, N. W. Winslow, and A. S. Kofford, "Topographic effects on infrasound propagation", J. Acoust. Soc. Am., 131(1), $35-46(\mathbf{2 0 1 2})$.

31. M. H. McKenna, B. W. Stump, and C. Hayward, "Effect of time-varying tropospheric models on near-regional and regional infrasound propagation as constrained by observational data", J. Geophys. Res., 113, D11111 (2008).

32. J. W. Melgarejo, and J. W. Deardorff, "Stability functions for the boundary layer 
Damiens, Millet and Lott, J. Acoust. Soc. Am., p. 45 resistance laws based upon observed boundary layer heights", J. Atmos. Sci., 31, 13241333 (1974).

33. J. W. Miles, "On the stability of heterogeneous shear flows", J. Fluid Mech., 10(04), 496-508 (1961).

34. C. Millet, J. C. Robinet, and C. Roblin, "On using computational aeroacoustics for long-range propagation of infrasounds in realistic atmospheres", Geophys. Res. Let., $34(14)(2007)$

35. C. J. Nappo, H. Y. Chun, and H. J. Lee, "A parameterization of wave stress in the planetary boundary layer for use in mesoscale models", Atmos. Env., 38, 2665-2675 $(2004)$

36. P. Queney, "The problem of air flow over mountains:A summary of theoretical studies", Bull. Am. Meteorol. Soc., 29, 16-26 (1948).

37. H. Richner, and P. Hachler, "Understanding and forecasting alpine Foehn", Mountain weather research and forecasting: recent progress and current challenges, F. K. Chow, S. F. J. De Wekker, and B. J. Snyder, Springer Science and Business Media, 219-260 (2013). 
Damiens, Millet and Lott, J. Acoust. Soc. Am., p. 46

38. P. Seibert, "South Foehn studies since the alpex experiment", Meteorol. and Atmos. Phys., 43, 91-103 (1990).

39. R. B. Smith, "The influence of mountains on the atmosphere". Adv. Geophys., 21, 87-230 (1979).

40. R. B. Smith, B. K. Woods, J. Jensen, W. A. Cooper, J. D. Doyle, Q. Jiang, and V. Grubisic, "Mountain waves entering the stratosphere", J. Atmos. Sci., 65, 2543-2562 (2008).

41. J. Sun, D.H. Lenschow, S.P. Burns, R.M. Banta, R.K. Newsom, R. Coulter, S. Frasier, T. Ince, C. Nappo, B.B. Balsey, M. Jensen, L. Mahrt, D. Millar, and B. Skelly, "Atmospheric disturbances that generate intermittent turbulence in nocturnal boundary layers", Bound.-Layer Meteor, 110, 255-279 (2003).

42. L. C. Sutherland, and H. E. Bass, "Atmospheric absorption in the atmosphere up to 160 km", J. Acoust. Soc. Am., 115(3), 1012-1032 (2004).

43. K. Szuberla, J. V. Olson, and K. M. Arnoult, "Explosion localization via infrasound", J. Acoust. Soc. Am., 125(5), 112-116 (2009).

44. R. Waxler, "A vertical eigenfunction expansion for the propagation of sound in a 
Damiens, Millet and Lott, J. Acoust. Soc. Am., p. 47 downward-refracting atmosphere over a complex impedance plane", J. Acoust. Soc. Am., 112, 2540-2552 (2002).

45. R. Waxler, "Modal expansions for sound propagation in the nocturnal boundary layer", J. Acoust. Soc. Am., 115, 1437-1448 (2004).

46. R. Waxler, C. L. Talmadge, S. Dravida, and K. E. Gilbert, "The near-ground structure of the nocturnal sound field", J. Acoust. Soc. Am., 119, 86-95 (2006).

47. R. Waxler, K. E. Gilbert, and C. Talmadge, "A theoretical treatment of the long range propagation of impulsive signals under strongly ducted nocturnal conditions", J. Acoust. Soc. Am., 124, 2742-2754 (2008).

48. R. Waxler, J. Assink, and D. Velea, "Modal expansions for infrasound propagation and their implications for ground-to-ground propagation", J. Acoust. Soc. Am., 141, 1290-1307 (2017).

49. C. D. Whiteman, and J. D. Whiteman, "An historical climatology of damaging downslope windstorms at Boulder, Colorado", NOAA Tech. Report ERL336-APCL 35 (1974).

50. C. Whiteman, X. Bian, and S. Zhong, "Low-level jet climatology from enhanced rawinsonde observations at a site in the southern great plains", J. Appl. Meteorol., 36, 1363-1376 (1997). 